\title{
The Role of Calcium in Growth Induced by Indole-3-Acetic Acid and Gravity in the Leaf-Sheath Pulvinus of Oat (Avena sativa)
}

\author{
Thomas G. Brock, ${ }^{1, *}$ James Burg, ${ }^{1}$ Najati S. Ghosheh, ${ }^{2}$ and Peter B. Kaufman ${ }^{1}$ \\ ${ }^{1}$ Department of Biology, University of Michigan, Ann Arbor, Michigan 48109, USA; and ${ }^{2}$ Biology Department, Eastern Michigan \\ University, Ypsilanti, Michigan 48197, USA
}

Received November 4, 1991; accepted February 19, 1992

Abstract. Leaf-sheath pulvini of excised segments from oat (Avena sativa L.) were induced to grow by treatment with $10 \mu \mathrm{M}$ indole-3-acetic acid (IAA), gravistimulation, or both, and the effects of calcium, EGTA, and calcium channel blockers on growth were evaluated. Unilaterally applied calcium $\left(10 \mathrm{mM} \mathrm{CaCl}_{2}\right)$ significantly inhibited IAAinduced growth in upright pulvini but had no effect on growth induced by either gravity or gravity plus IAA. Calcium alone had no effect on upright pulvini. The calcium chelator EGTA alone $(10 \mathrm{mM})$ stimulated growth in upright pulvini. However, EGTA had no effect on either IAA- or gravityinduced growth but slightly diminished growth in IAA-treated gravistimulated pulvini. The calcium channel blockers lanthanum chloride $(25 \mathrm{mM})$, verapamil $(2.5 \mathrm{mM})$, and nifedipine $(2.5 \mathrm{mM})$ greatly inhibited growth as induced by IAA $(\geqslant 50 \%$ inhibition) or IAA plus gravity ( $20 \%$ inhibition) but had no effect on gravistimulated pulvini. Combinations of channel blockers were similar in effect on IAA action as individual blockers. Since neither calcium ions nor EGTA significantly affected the graviresponse of pulvini, we conclude that apoplastic calcium is unimportant in leaf-sheath pulvinus gravitropism. The observation that calcium ions and calcium channel blockers inhibit IAA-induced growth, but have no effect on gravistimulated pulvini, further supports previous observations that gravistimulation alters the responsiveness of pulvini to IAA.

Consistent with the classical Cholodny-Went model (Cholodny 1926, Went 1926), as well as contempo-

\footnotetext{
* Present address: Department of Pediatrics, 7500 Medical Science Research Building I, University of Michigan, Ann Arbor, MI 48109-0648, USA.
}

rary theory (Kaufman and Song 1987, Moore and Evans 1986, Pickard 1985), gravitropism consists of three components: stimulus perception, transduction of this perceived signal, and the asymmetric growth response. In shoots of festucoid grasses like oat and barley, a single organ, the leaf-sheath pulvinus, is the site of both perception (Brock and Kaufman 1988c, Song et al. 1988) and response (Brock et al. 1989). However, the pulvinus shows no graviresponse when physically isolated from the rest of the plant (Brock et al. 1991), suggesting that other tissues are required for transduction.

Calcium ions have been implicated in the gravitropism of several plant organs, including maize roots (Lee et al. 1983a,b), pea roots (Lee et al. 1984), sunflower hypocotyls (Arslan-Cerim 1966, Goswami and Audus 1976), oat coleoptiles (Slocum and Roux 1983), and maize coleoptiles (Goswami and Audus 1976). The function of calcium ions in gravitropism is unclear. In the most striking case, calcium applied asymmetrically to the root cap of maize induces tropic-like curvature (Lee et al. 1983b). Calcium chelating agents applied to root caps inhibit gravitropism (Lee et al. 1983b), suggesting that extracellular calcium plays a critical role in the tropic response. Since the root cap is recognized as the site of graviperception, the apoplastic calcium may be active during the stimulus perception phase of gravitropism. However, calcium movement within the root cap is linked to a redistribution in the opposite direction of the growth-regulating hormone indole-3-acetic acid (IAA) (Lee et al. 1984). As a result, calcium may function in the transduction phase of gravitropism (Evans et al. 1986).

Furthermore, calcium redistribution occurs in the elongation zone of maize roots (Lee and Evans 1985) and oat coleoptiles (Slocum and Roux 1983). Similarly, inhibitors of the calcium-modulated pro- 
tein calmodulin inhibit the graviresponse in the elongation zone of oat coleoptiles (Biro et al. 1982). Since the elongation zone is the site of the graviresponse, calcium may be active in this phase of gravitropism as well.

In this study, we assess the role of calcium in the graviresponse of the leaf-sheath pulvinus of oat (Avena sativa L.). Specifically, we evaluate the effects of increased and decreased apoplastic calcium, as well as the inhibition of calcium uptake via calcium-specific channels, on pulvinus growth. These effects are tested on pulvini induced to grow by either the hormone IAA or gravistimulation, or both.

\section{Materials and Methods}

Oat plants (Avena sativa L. cv. Victory, from Svenska Allmanna A.B., Svalof, Sweden) were grown under greenhouse conditions, as described previously (Brock and Kaufman 1988a). When plants were approximately 45 days old, individuals were selected for length of next-to-last internode between 4 and $10 \mathrm{~cm}$ (for anatomical detail, see Brock and Kaufman 1990, Kaufman et al. 1987). At this stage, the next-to-last pulvinus was maximally graviresponsive (Brock and Kaufman 1988b).

For experimentation, stem sections were selected for straightness of stem and uniformity of pulvinus size, then excised $4 \mathrm{~cm}$ below and $4 \mathrm{~cm}$ above the next-to-last pulvinus and placed upright in double-distilled water containing $0.1 \mathrm{M}$ sucrose. Pulvini were then lightly abraded on all sides with jeweler's rouge to reduce the effectiveness of the cuticle as a barrier to solute movement. In preliminary experiments, the abrasion treatment did not alter the graviresponse of pulvini (Brock and Kaufman 1988a). However, this treatment did alter the magnitude of response to applied chemicals, although responses were not changed qualitatively. Abrasion also reduced the variation in the magnitude of response to applied agents (Brock and Kaufman 1988a).

All chemicals were prepared and applied in $1 \%$ agar $(\mathrm{w} / \mathrm{v})$ with $\mathrm{pH}$ adjusted to $5.5 \pm 0.1$ with $10 \mathrm{mM}$ Hepes $/ \mathrm{HCl}$ buffer. In some experiments, segments were pretreated for $30 \mathrm{~min}$ in a vertical orientation. Final concentrations were as follows: IAA at $10 \mu \mathrm{M}$; $\mathrm{CaCl}_{2}$ and EGTA at $10 \mathrm{mM} ; \mathrm{LaCl}_{3}$ at $25 \mathrm{mM}$; verapamil and nifedipine at $2.5 \mathrm{mM}$. All chemicals were purchased from Sigma Chemical Co. (St. Louis, MO, USA).

Excised segments were marked on one side with a fine tip Sharpie and measured for initial angle (typically between $0-10^{\circ}$ ), then secured below the pulvinus to Plexiglas supports, with segment bases in 0.1 M sucrose (Brock and Kaufman 1988a). Agar blocks were applied unilaterally to abraded pulvini, with agar block volume being at least 12 times the pulvinus volume. Segments were then left upright (i.e., not gravistimulated) or placed horizontal (gravistimulated) in the dark in humid chambers at 25 $\pm 1^{\circ} \mathrm{C}$ for $24 \mathrm{~h}$. In gravistimulated treatments, the agar block touched the lower face of the pulvinus.

Graviresponse was measured as the change in angle through the pulvinus and also as the change in lengths of the upper and lower (or untreated and treated) sides of the pulvinus over the 24 $h$ interval. Here, graviresponse is presented only as the change in angle through the pulvinus over the $24 \mathrm{~h}$ interval. This parameter was chosen as it gave a more sensitive and readily measured

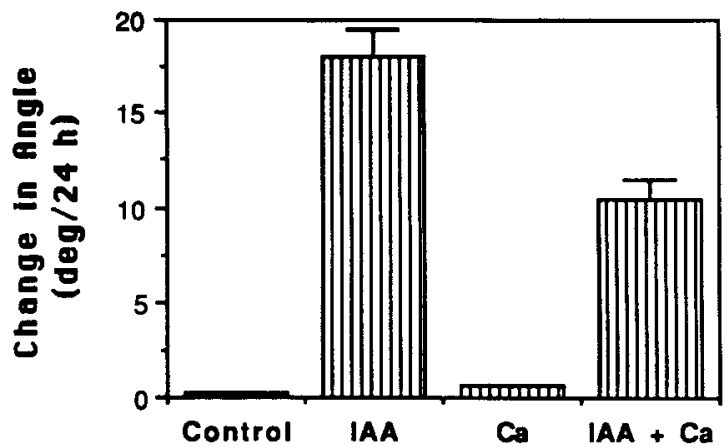

Fig. 1. Effect of IAA and calcium on pulvinus growth in upright segments. Agar blocks $(1 \% \mathrm{w} / \mathrm{v})$ containing IAA $(10 \mu \mathrm{M})$ and $/$ or $\mathrm{CaCl}_{2}(10 \mathrm{mM})$ were applied unilaterally to abraded pulvini in vertically oriented segments placed in $0.1 \mathrm{M}$ sucrose in the dark. After $24 \mathrm{~h}$, the change in angle through the pulvinus was used as a measure of the pulvinus growth response. Bars indicate SE.

indication of response than a direct measurement of length of the pulvinus. In every case, change in angle resulted primarily from an increase in length of the lower (or treated) side of the pulvinus.

All experiments were repeated at least three times. Statistical significance was evaluated using either the nonparametric MannWhitney $U$-test, with $p=0.05$, or the Student's $t$-test with a level of significance of 0.05 , as appropriate. Sample size ranged from 5-20 pulvinus-containing stem segments per treatment per experiment.

\section{Results}

When stem segments were left in a vertical orientation, calcium alone had no effect on the pulvinus (Fig. 1). As noted previously (Dayanandan et al. 1977, Maeda 1958, Osborne and Wright 1977, Wright et al. 1978), IAA strongly promotes pulvinus growth in length in upright pulvini. Calcium significantly inhibited this effect.

In contrast, pulvinus growth, represented as the change in angle through the pulvinus, was not significantly affected by $10 \mu \mathrm{M}$ IAA (Fig. 2). This was consistent with previously published results (Brock and Kaufman 1988a) and is indicative of the change in responsiveness to IAA observed in pulvini upon gravistimulation. Also, neither calcium alone nor IAA plus calcium affected the graviresponse of pulvini.

The calcium chelator EGTA significantly stimulated the growth of ungravistimulated pulvini, to a small degree (Fig. 3). This result was observed repeatedly and consistently. The effect of IAA + EGTA was similar to that of IAA alone in upright segments.

When pulvini were gravistimulated, EGTA had 


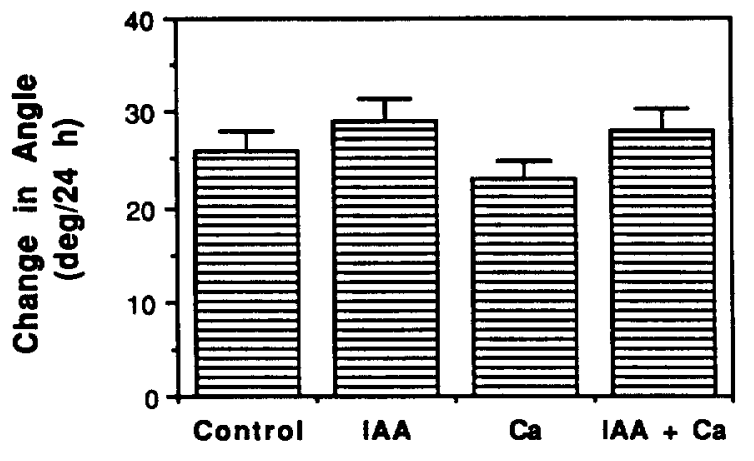

Fig. 2. Effect of IAA and calcium on pulvinus growth in gravistimulated segments. Conditions as described in Fig. 1, except that the agar blocks contacted the lowermost side of pulvini in horizontally placed segments. Bars indicate SE.

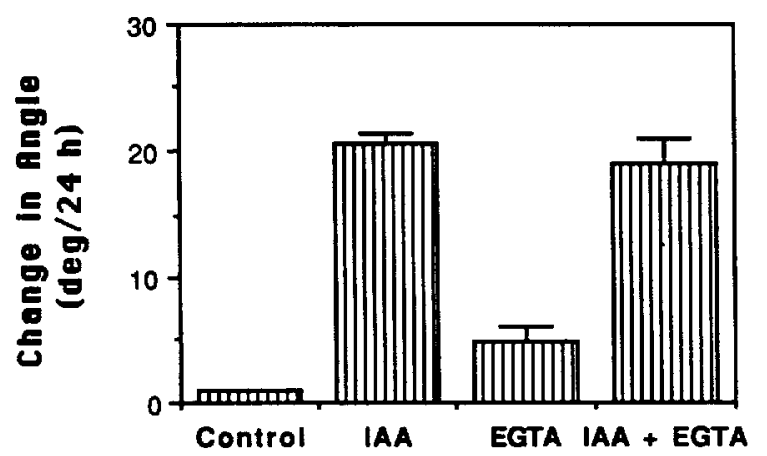

Fig. 3. Effect of IAA and EGTA $(10 \mathrm{mM})$ on pulvinus growth in upright segments. Conditions as described in Fig. 1.

no effect by itself (Fig. 4). However, the response of gravistimulated pulvini to IAA + EGTA was consistently less than that due to IAA alone.

The three calcium channel blockers-lanthanum, verapamil and nifedipine-had similar effects. None stimulated growth of pulvini from upright segments (Fig. 5). In vertically oriented pulvini, all channel blockers significantly inhibited the response to IAA, with inhibition commonly greater than $50 \%$.

When pulvini were gravistimulated, IAA at 10 $\mu \mathrm{M}$ had little effect on the gravity-induced pulvinus growth, as seen previously (Fig. 6). Lanthanum typically reduced the gravity-induced growth only minimally, and this change was rarely statistically significant. However, treatment of gravistimulated pulvini with IAA plus lanthanum resulted in a statistically significant reduced growth response. Similar patterns of response were also observed when verapamil or nifedipine were used in place of lanthanum.

Although all three channel blockers produced

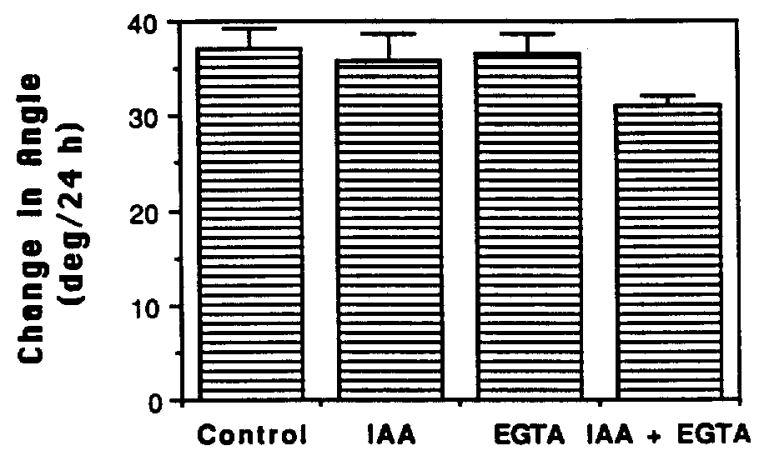

Fig. 4. Effect of IAA and EGTA on pulvinus growth in gravistimulated segments. Conditions as described in Fig. 2.

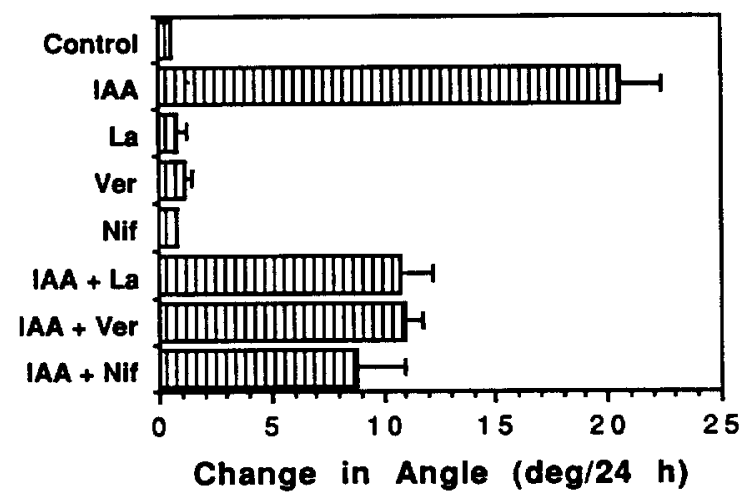

Fig. 5. Effect of IAA and calcium channel blockers on pulvinus growth in upright segments. The calcium channel blockers lanthanum chloride $(25 \mathrm{mM})$, verapamil $(2.5 \mathrm{mM})$, and nifedipine $(2.5 \mathrm{mM})$ were prepared in agar blocks with or without IAA and applied unilaterally to pulvini in vertically oriented segments.

similar results, it is possible that their effects were not expressed through identical mechanisms. To test this, pairs of blockers were tested together for effectiveness. Treatment of upright pulvini with both lanthanum and verapamil resulted in responses that were similar to either alone: lanthanum plus verapamil did not induce a growth response alone but did significantly inhibit IAA action (Fig. 7). Other combinations of the three channel blockers, at various concentrations, did not significantly change the effect of individual blockers on IAA action in upright pulvini.

\section{Discussion}

Several details relevant to pulvinus gravitropism are apparent from these results. First, the physiology of the gravitropic response in the leaf-sheath pulvinus is not like that of the other well-studied 


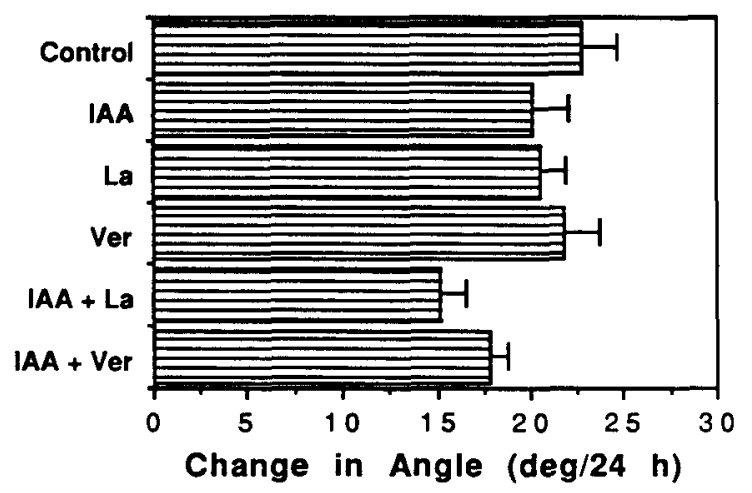

Fig. 6. Effect of IAA and calcium channel blockers on pulvinus growth in gravistimulated segments. Channel blockers were applied, as described in Fig. 5, to the lowermost side of gravistimulated pulvini.

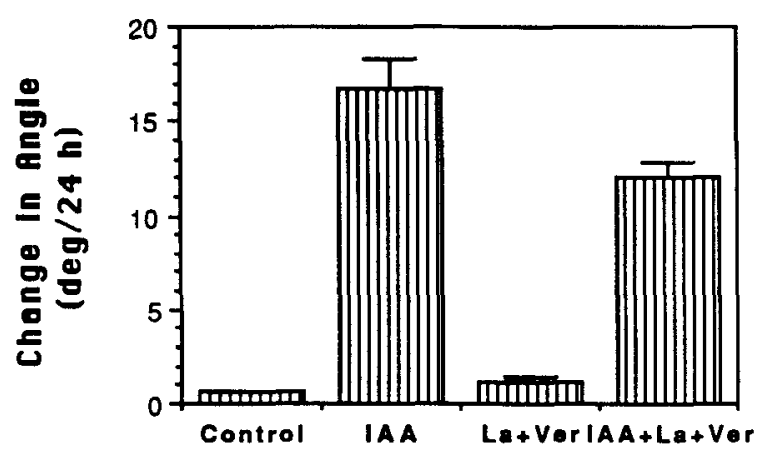

Fig. 7. The effect of lanthanum plus verapamil on pulvinus growth, with or without IAA, in vertically oriented segments. Treatments with channel blockers and IAA were as described in Fig. 5.

model of gravitropism, the maize root tip. For example, in the maize root tip, asymmetric distribution of calcium induces asymmetric growth (Lee et al. 1983a,b), whereas it has no effect in the leafsheath pulvinus (Fig. 1). Also, calcium channel blockers completely inhibit the graviresponse of the maize root tip (Stinemetz and Evans 1986); however, they have only a modest effect on the oat leaf-sheath pulvinus (Fig. 6). This difference in physiology should not be surprising, considering the differences in the multiple functions of the two organs. Specifically, the functions of the root tip include gravitropism, water and nutrient sensing and accumulation, and water, nutrient, and mechanical stress avoidance. The pulvinus, in contrast, appears to function largely as a graviresponding organ, with a minor role in the mechanical support of the shoot. These differences in function will necessarily be associated with differences in physiology.
Second, apoplastic calcium plays a minimal role in the gravitropism of the pulvinus. Neither elevation of extracellular calcium, nor calcium depletion via EGTA treatment, significantly alter the graviresponse. Since a normal graviresponse indicates successful graviperception and transduction of the signal, apoplastic calcium is not critical in these steps as well.

In some systems (e.g., maize root tips, sunflower hypocotyls), calcium and IAA interact in an antiport fashion: calcium movement in one direction is tightly linked to IAA movement in the opposite direction (De Guzman and Dela Fuente 1984, Dela Fuente 1984). Perturbing either one affects the other, and this can alter the graviresponse. In the gravistimulated pulvinus, however, lateral auxin movement occurs but is not necessary for a graviresponse (Brock et al. 1991). If calcium influences auxin movement in the leaf-sheath pulvinus, it was not evident from these experiments.

Third, calcium channels play a major role in IAAinduced pulvinus growth, but are less essential to gravity-induced growth. Channel blockers greatly inhibit IAA action, more so in upright pulvini than in gravistimulated ones. Yet these blockers have little effect on gravistimulated pulvini in the absence of IAA. Hence, gravistimulation mollifies the effect of the channel blockers. A similar argument holds for extracellular calcium, which is more striking since gravistimulation eliminates its inhibitory effect on IAA.

Clearly, and most importantly, gravistimulation has effects on pulvinus physiology that are distinct from the effects of IAA. That is, transduction of the perceived gravistimulus leads to significant cellular changes that are distinct from simple changes in IAA dynamics: these are gravity-specific, hormoneindependent changes. This is consistent with the observation that gravistimulation alters the responsiveness of pulvini to applied IAA and gibberellic acid (Brock and Kaufman 1988a). The challenge will be to elucidate the nature of these changes.

Acknowledgments. The technical assistance of Ms. Virginia Bright is gratefully acknowledged. Thanks also to Carol M. Fast for her support and invaluable contributions, and to Peter Thomas and Allison Elizabeth for technical assistance.

\section{References}

Arslan-Cerim N (1966) The redistribution of radioactivity in geotropically stimulated hypocotyls of Helianthus annuus pretreated with radioactive calcium. J Exp Bot 17:236-240

Biro RL, Hale CC, Wiegand OF, Roux SJ (1982) Effects of chlor- 
promazine on gravitropism in Avena coleoptiles. Ann Bot 50:737-745

Brock TG, Kapen EH, Ghosheh NS, Kaufman PB (1991) Dynamics of auxin movement in the gravistimulated leafsheath pulvinus of oat (Avena sativa). J Plant Physiol 138:57-62

Brock TG, Kaufman PB (1988a) Altered growth response to exogenous auxin and gibberellic acid by gravistimulation in pulvini of Avena sativa. Plant Physiol 87:130-133

Brock TG, Kaufman PB (1988b) Competency for graviresponse in the leaf-sheath pulvinus of Avena sativa: Onset to loss. Amer J Bot 75:1672-1677

Brock TG, Kaufman PB (1988c) Effect of dark pretreatment on the kinetics of response of barley pulvini to gravistimulation and hormones. Plant Physiol 88:10-12

Brock TG, Kaufman PB (1990) Movement in grass shoots. In: Satter RL, Gorton HL, Vogelmann TC (eds) The pulvinus: Motor organ for leaf movement. Academic Press, New York, pp 59-71

Brock TG, Lu CR, Ghosheh NS, Kaufman PB (1989) Localization and pattern of graviresponse across the pulvinus of barley (Hordeum vulgare). Plant Physiol 91:744-748

Cholodny N (1926) Beitrage zur Analyse der geotropischen Reaktion. Jahrb Wiss Bot 65:447-459

Dayanandan P, Hebard FV, Baldwin VD, Kaufman PB (1977) Structure of gravity-sensitive sheath and internodal pulvini in grass shoots. Amer J Bot 64:1189-1199

De Guzman CC, Dela Fuente RK (1984) Polar calcium flux in sunflower hypocotyl segments. I. The effect of auxin. Plant Physiol 76:347-352

Dela Fuente RK (1984) Role of calcium in the polar secretion of indoleacetic acid. Plant Physiol 76:342-346

Evans ML, Moore R, Hasenstein K-H (1986) How roots respond to gravity. Sci Amer 254:112-118

Goswami KKA, Audus LJ (1976) Distribution of calcium, potassium and phosphorous in Helianthus annuus hypocotyls and Zea mays coleoptiles in relation to tropic stimuli and curvatures. Ann Bot 40:49-64

Kaufman PB, Brock TG, Song I, Rho YB, Ghosheh NS (1987) How cereal grass shoots perceive and respond to gravity. Amer J Bot 74:1446-1457

Kaufman PB, Song I (1987) Hormones and the orientation of growth. In: Davies PJ (ed) Plant hormones and their role in plant growth and development. Martinus Nijhoff Publishers, Dordrecht, pp 375-392

Lee JS, Evans ML (1985) Polar transport of auxin across gravistimulated roots and maize and its enhancement by calcium. Plant Physiol 77:824-827

Lee JS, Mulkey TJ, Evans ML (1983a) Gravity-induced polar transport of calcium across root tips of maize. Plant Physiol 73:874-876

Lee JS, Mulkey TJ, Evans ML (1983b) Reversible loss of gravitropic sensitivity in maize roots after tip application of calcium chelators. Science 220:1375-1376

Lee JS, Mulkey TJ, Evans ML (1984) Inhibition of polar calcium movement and gravitropism in roots treated with auxintransport inhibitors. Planta 160:536-543

Maeda I (1958) The effects of growth regulators on the geotropism of the leaf sheath basal region in wheat. J Exp Bot 9:343-349

Moore R, Evans ML (1986) How roots perceive and respond to gravity. Amer J Bot 73:574-587

Osborne DJ, Wright M (1977) Gravity-induced cell elongation. Proc Royal Soc London Ser B 199:551-564

Pickard B (1985) Role of hormones, protons and calcium in geotropism. In: Pharis RP, Reid DM (eds) Encyclopedia of plant physiology (new series), vol 11. Springer-Verlag, Berlin, pp 179-261

Slocum RD, Roux SJ (1983) Cellular and subcellular localization of calcium in gravistimulated oat coleoptiles and its possible significance in the establishment of tropic curvature. Planta 157:481-492

Song I, Lu CR, Brock TG, Kaufman PB (1988) Do starch statoliths act as the gravisensors in cereal grass pulvini? Plant Physiol 86:1155-1162

Stinemetz CL, Evans ML (1986) The effect of calmodulin antagonists on the asymmetric redistribution of $\mathrm{Ca}^{++}$and IAA following gravistimulation. Proc Amer Soc Grav and Space Biol, 2nd Annual Symposium of the NASA Space Biology Program, Charlottesville, VA, p 9

Went FW, Thimann KV (1926) Phytohormones. Macmillan, New York

Wright M, Mousdale DMA, Osborne DJ (1978) Evidence for a gravity regulated level of endogenous auxin controlling cell elongation and ethylene production during geotropic bending in grass nodes. Biochem Physiol Pflanzen 172:581-596 